Check for updates

Cite this: Nanoscale, 2021, 13, 5224

Received 23rd December 2020,

Accepted 13th February 2021

DOI: 10.1039/d0nr09075a

rsc.li/nanoscale

\section{Structural insights into fusion mechanisms of small extracellular vesicles with model plasma membranes $\uparrow$}

\author{
Fabio Perissinotto, (iD $\ddagger^{a}, \mathrm{~b}$ Valeria Rondelli, (D) $\mathbf{t}^{\mathrm{c}}$ Beatrice Senigagliesi, ${ }^{a}$ Paola Brocca, ${ }^{c}$

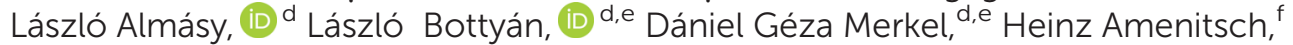 \\ Barbara Sartori, ${ }^{f}$ Karin Pachler, ${ }^{\text {g,h }}$ Magdalena Mayr, ${ }^{g}$ Mario Gimona, ${ }^{\text {g,h }}$ Eva Rohde, ${ }^{\text {, }, i}$ \\ Loredana Casalis (iD *a and Pietro Parisse (iD *a,j
}

\begin{abstract}
Extracellular vesicles (EVs) are a potent intercellular communication system. Such small vesicles transport biomolecules between cells and throughout the body, strongly influencing the fate of recipient cells. Due to their specific biological functions they have been proposed as biomarkers for various diseases and as optimal candidates for therapeutic applications. Despite their extreme biological relevance, their mechanisms of interaction with the membranes of recipient cells are still hotly debated. Here, we propose a multiscale investigation based on atomic force microscopy, small angle X-ray scattering, small angle neutron scattering and neutron reflectometry to reveal structure-function correlations of purified EVs in interaction with model membrane systems of variable complex compositions and to spot the role of different membrane phases on the vesicle internalization routes. Our analysis reveals strong interactions of EVs with the model membranes and preferentially with the borders of protruding phase domains. Moreover, we found that upon vesicle breaking on the model membrane surface, the biomolecules carried by/on EVs diffuse with different
\end{abstract}

\footnotetext{
${ }^{a}$ Elettra Sincrotrone Trieste, Trieste, Italy. E-mail: Pietro.parisse@elettra.eu, Loredana.casalis@elettra.eu

${ }^{b}$ Center for Infection and Immunity of Lille, INSERM U1019, Institut Pasteur de Lille, Lille, France

${ }^{c}$ Department of Medical Biotechnology and Translational Medicine, Università degli Studi di Milano, Italy

${ }^{d}$ Centre for Energy Research, Budapest, Hungary

${ }^{e}$ Wigner Research Centre for Physics, Budapest, Hungary

${ }^{f}$ Institute of Inorganic Chemistry, Graz University of Technology, Graz, Austria

${ }^{g}{ }_{G M P}$ Unit, Spinal Cord Injury and Tissue Regeneration Center Salzburg, Paracelsus Medical University (PMU), Salzburg, Austria

${ }^{h}$ Research Program "Nanovesicular Therapies", Paracelsus Medical University, Salzburg, Austria

${ }^{i}$ Department of Transfusion Medicine, University Hospital, Salzburger Landeskliniken, Austria

${ }^{j}$ CNR-IOM, Trieste, Italy

$\dagger$ Electronic supplementary information (ESI) available: Materials and methods, sEV scattering length density from SAXS, neutron reflectometry data, AFM analysis of partial DOPC SLBs, proteinase K experiments, sEVs' island coverage evolution during time. See DOI: 10.1039/d0nr09075a

$\$$ These two authors contributed equally to this work.
}

kinetics rates, in a process distinct from simple fusion. The biophysical platform proposed here has clear implications on the modulation of EV internalization routes by targeting specific domains at the plasma cell membrane and, as a consequence, on EV-based therapies.

\section{Introduction}

Extracellular vesicles (EVs) are nanosized, cell-derived vesicles devoted to the transport of macromolecules, metabolites and nutrients throughout the body. In the last 15 years, they received increasing attention due to their fundamental role in intercellular communication. ${ }^{1}$ EVs are ubiquitously involved in most physiologically relevant processes and according to their biogenesis, size, structure and composition, they can be classified into three main classes: exosomes, microvesicles and apoptotic bodies. Conventional isolation methods enrich vesicles of different sizes. Herein we will follow the terminology as suggested in the MISEV 2018 guidelines and refer to small EV (sEVs) and large EVs (LEVs). ${ }^{2}$ Notably, they contain specific signatures from the originating cells and can strongly influence the fate of recipient cells. Hence, EVs have been proposed as biomarkers in several diseases. ${ }^{3-6}$ Moreover, their natural biocompatibility, biological function and small size make them optimal candidates as therapeutic agents in several frameworks ranging from immune therapy to vaccination and from regenerative medicine to drug delivery. ${ }^{7,8}$ However, despite their recognized biomedical relevance, the field is not yet fully mature and more in-depth studies are required to understand EV physiology. In particular, the correlation of biophysical and biochemical properties of isolated EV subpopulations with their biological function is under continuous debate, also due to the difficulty in establishing an accepted standard for isolation processes, which can influence the EVs' properties. ${ }^{9-11}$ Moreover, an overall understanding of cell internalization mechanisms of EVs is still lacking. ${ }^{12-15}$ The nanoscale spatio- 
temporal details on how EVs interact, adsorb, and fuse with target cells, as well as the factors influencing the biogenesis and release of the molecular cargo, although crucial to devise EV-based therapies, still lack a comprehensive description. The literature suggests a wide variety of routes for cellular uptake, ${ }^{14-17}$ depending on the specific composition of the cellular membrane, EV function(s) and their physico-chemical properties. ${ }^{17-19}$ It is expected that uptake dynamics and membrane fusion mechanisms are tightly related to the potency and function of EVs and are found to play a key role in EVbased drug delivery applications. ${ }^{20}$ Uptake dynamics in turn has been shown to depend on EV size ${ }^{21}$ and on the extracellular matrix environment, ${ }^{22}$ but results on fixed and live cells are quite scattered. ${ }^{23}$ In order to elucidate the EV/recipient cell interactions, here we exploit artificial lipid membranes as tunable model platforms to mimic natural cell membranes. ${ }^{23-27}$ In particular, we challenge our experiments to quantify the dynamics of interaction between fully characterized small EVs and model membranes to reveal the relationship between function and biophysical properties of these vesicles. Standardized protocols and good manufacturing practice conditions were employed to derive highly stable vesicles of defined size and reproducible molecular profiles from umbilical cord multipotent Mesenchymal Stem (Stromal) Cells (MSCs). After a thorough biophysical and biochemical characterization of EVs, non-contact liquid imaging Atomic Force Microscopy (AFM) and, in parallel, Neutron Reflectometry (NR), as well as Small Angle Neutron Scattering (SANS) experiments were performed on EVs to determine their interaction with lipid bilayers. While AFM provides information on the topography of the outermost surface of the membrane with sub-nanometer resolution, in the physiological environment, neutron scattering yields the contrast to address the role of specific lipid component in the interaction, with structural information extending over the entire lipid bilayer transversal section. As a start, we focused on complex 3 component (phospholipids, sphingolipids and cholesterol) synthetic membranes via AFM imaging, to investigate specific sEVs-to-membrane domain interactions. Then, having identified the preferential interaction lipid domain, we focused on the simple membrane made of the specific single phase, to exploit an integrated morphological/structural experimental approach providing crucial information on the mechanisms of EV internalization, as the partial fusion of the EVs with the model membrane bilayer. Our approach and findings pave the way for identifying specific vesicle-cell uptake routes and for eventually tuning them for therapeutic needs.

\section{Results and discussion}

Efficient isolation of small EVs (sEVs) is the first mandatory step to assure reliable results. A plethora of isolation protocols has been reported in the literature, with pros and cons depending on the specific system in use. ${ }^{28}$ Here, we isolated sEVs from the umbilical cord MSC conditioned medium (fol- lowing the protocol reported in the ESI $\dagger$ ) and further characterized them with different, complementary techniques. Nanoparticle tracking analysis (NTA) shows that sEV preparations contain particles with a mean size of $120 \pm 50 \mathrm{~nm}$ (Fig. 1a). As confirmed by the clear visualisation of vesicle membranes in the cryo-EM images (Fig. 1c), these preparations include vesicles. This finding is further supported by the presence of typical sEV/exosome markers, such as tetraspanins CD9, CD63 and CD81 in the Multiplex bead-based flow cytometry assay ${ }^{29}$ profiles (Fig. 1b). ${ }^{30}$ AFM profiles from the topographic images in liquid (Fig. 1d and e) confirmed the size distribution of sEVs observed by NTA and cryo-EM analysis, highlighting the presence of particles with size less than $50 \mathrm{~nm}$, not detectable by NTA. In Fig. 1e, the vesicles are shown to exhibit equivalent diameters ranging between 30 and $150 \mathrm{~nm}$ (mean value \pm standard deviation: $60 \pm 30 \mathrm{~nm}$ ) and heights ranging from 10 to $80 \mathrm{~nm}$ (mean value \pm standard deviation: $30 \pm 15 \mathrm{~nm}$ ). The slight deformation with respect to their supposed spherical shape may be attributed to the small force applied by the AFM tip, the immobilization on the mica surface and possible tip convolution effects. ${ }^{31-33}$ To gain further information on the structural properties of sEVs, we performed Small Angle X-ray Scattering (SAXS) and SANS experiments on sEVs in solution (Fig. 1f). Analogous approaches have been applied to investigate similar systems. ${ }^{34-36}$ The SAXS profile in the investigated $q$-range, analysed by a simple core multi-shell model, is consistent with an asymmetric membrane profile, reported in Fig. S1, $\dagger$ accounting for extended polar components. However, the detailed feature at $q$ values around $0.06-0.08 \AA^{-1}$ may originate from some characteristic distance in the range of $10 \mathrm{~nm}$ occurring among objects on the membrane surface, that might also explain the average pattern of rugosity observed by AFM (Fig. 1). Again, the same pattern might be responsible for the deviation of the detected SANS intensity (in the analogous $q$-vector range) from the scattering profile of the $2 \mathrm{~nm}$ thick shell corresponding to the membrane core (fitting curve in Fig. 1f).

To further characterize sEVs, we performed NR studies. As a sample, we used the double-layer lipid film originated from the deposition and fusion of single sEVs on the surface of a macroscopic silicon support, covering it up to $90 \%$ of the total surface. A layer of $0.5 \mathrm{~nm}$ water was measured between the membrane and the silicon support. The cross-sectional structure of the supported bilayer probed by NR, in analogy with what was already performed on other model membranes as well as bacteria extract systems, ${ }^{37,38}$ is shown in Fig. S2 $\dagger$ together with its best fit (parameters in Table S1†), while in Fig. 1g, the corresponding scattering length density (SLD) profiles are reported. NR data on SEVs with two different solvent contrasts were collected and simultaneously fitted (Fig. 1g, $\mathrm{D}_{2} \mathrm{O}$, blue line; $\mathrm{H}_{2} \mathrm{O}$ yellow line). Data analysis confirmed complete fusion of sEVs on the silicon surface giving rise to a membrane of $6.9 \pm 0.2 \mathrm{~nm}$ thickness, with a SLD of $2.5 \pm 0.2 \times$ $10^{-6} \AA^{-2}$. Considering a mean SLD for fluid lipids of $-0.2 \times$ $10^{-6} \AA^{-2}$ [calculated for a $60: 40 \mathrm{~mol}$ : mol mixture of acyl 
a)

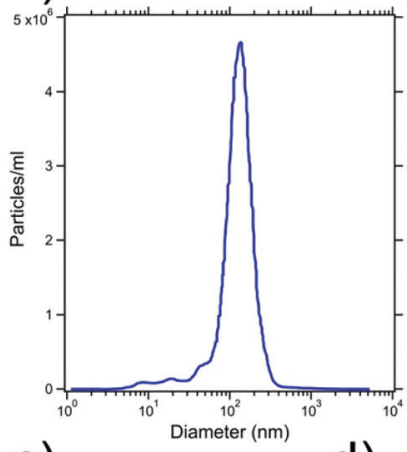

c)

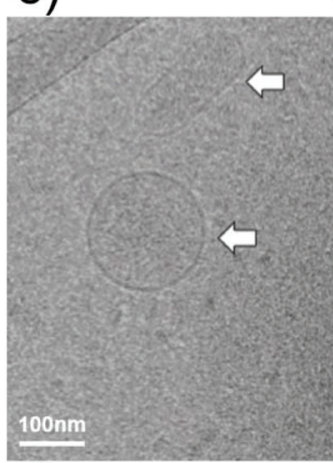

f)

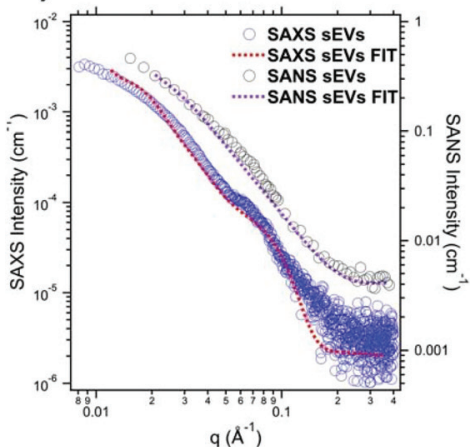

b)

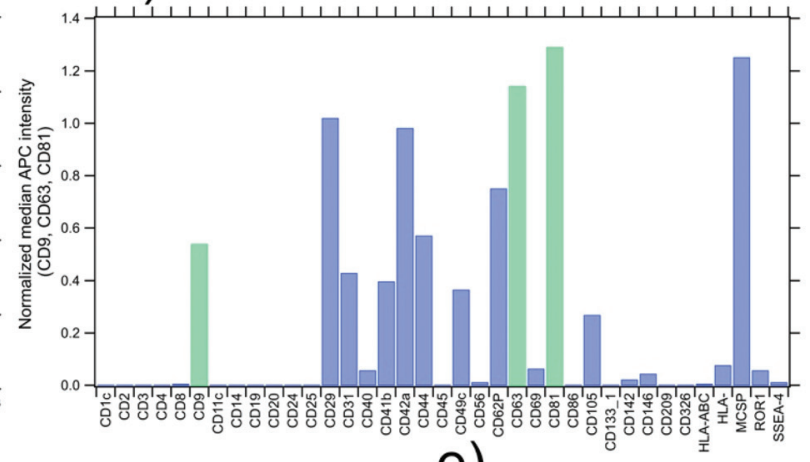

e)

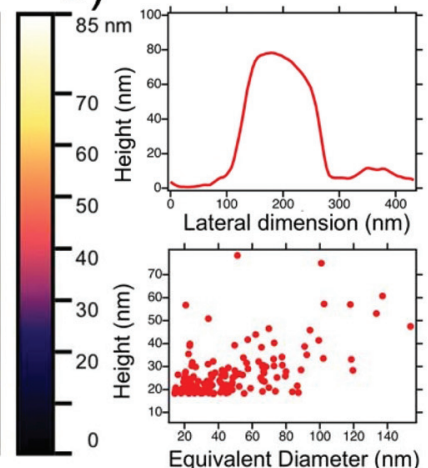

g)
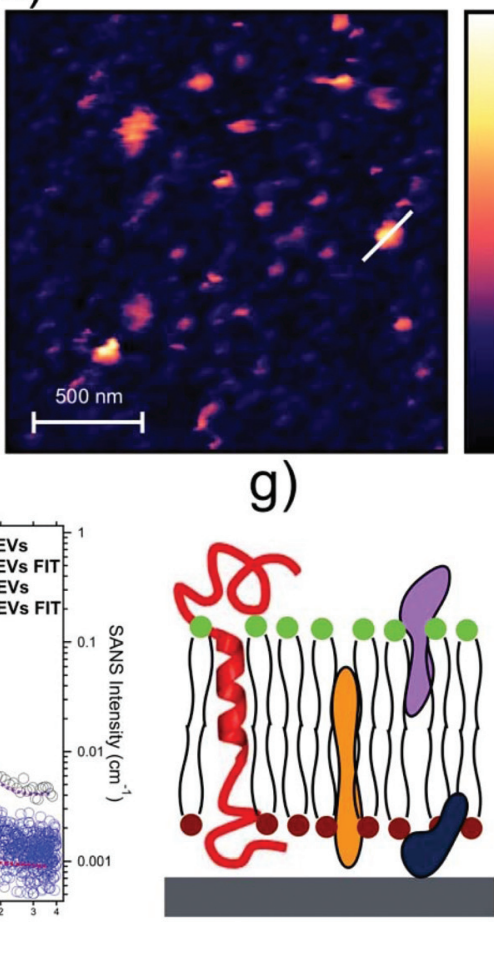

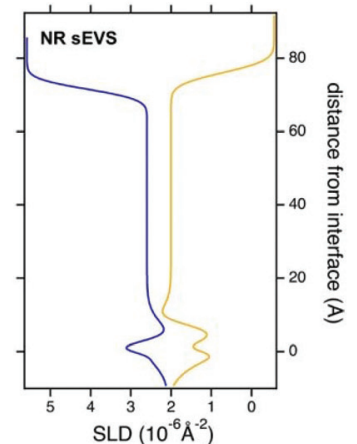

Fig. 1 Characterization of isolated small extracellular vesicles. (a) Size distribution of MSC-derived sEVs by nanoparticle tracking analysis. (b) Surface marker profiling by MACSPlex. Standard EV markers CD9, CD63 and CD81 are marked in green. (c) Cryo-electron microscopy image from a representative batch of MSC-derived SEVs isolated as described in the Methods section. The lipid bilayer surrounding the sEV can be unambiguously recognized (arrows). ( $d$ and e) AFM topographic image and corresponding line profile (upper panel) of MSC-sEVs deposited on the mica surface. Image acquired in PBS. (e, bottom panel) Correlation diagram between the equivalent diameter and the height extracted from AFM analysis. (f) SANS (black circles) and SAXS (blue circles) profiles of MSC-derived SEVs and corresponding fits (dark pink dotted line and red dotted line) with a coreshell model. (g) Scattering length density profiles of a sEV-derived supported lipid bilayer (SLB) in $\mathrm{H}_{2} \mathrm{O}$ (orange) and $\mathrm{D}_{2} \mathrm{O}$ (blue) obtained by neutron reflectivity with a pictorial sketch to help in SLD profile interpretation. Measured reflectivity data and best fit parameters are reported in the ESI (Table S1†).

chains $\left(-0.3 \times 10^{-6} \AA^{-2}\right)$ and cholesterol $\left.\left(0.2 \times 10^{-6} \AA^{-2}\right)\right]$ and of $2.5 \times 10^{-6} \AA^{-2}$ for the proteins, an estimation of the lipid to non-lipid ratio of 22 to 78 (by volume) is obtained. These values are in agreement with the estimated value found in the literature of roughly $50 \%$ molar of lipids. ${ }^{39}$ Regarding the sEVbased bilayer measured thickness $(6.9 \pm 0.2 \mathrm{~nm})$, although higher than the case of both synthetic and natural lipid extracts which is generally around $5.5 \mathrm{~nm},{ }^{40-42}$ we can say that it is compatible with one single bilayer and consistent with the presence of molecules other than lipids, as large proteins, originating from the SEV deposited membrane. The system interfacial roughness was found to be as low as $0.6 \pm 0.2 \mathrm{~nm}$. Overall, this analysis confirms a high lateral homogeneity which gives further strength to the entire approach and provides a strong ground to the data analysis model confirming a successful deposition of sEV.

We then proceeded to investigate the interaction of sEVs with planar supported phospholipid bilayers (SLB) that we pre- 
pared and characterized by thorough AFM topographic imaging and used as model plasma membranes. To address real membrane complexity, we prepared mixed bilayers composed of DOPC : sphingomyelin (SM) 2:1 with 5\% cholesterol. According to the relative three-component phase diagram, ${ }^{43}$ such mixed SLBs present, at room temperature, a phase separation between domains in the liquid ordered (Lo) phase enriched in SM and domains in the liquid disordered (Ld) phase enriched in PC. ${ }^{44}$

AFM analysis reported in Fig. 2a shows the presence of domains protruding $1.5 \mathrm{~nm}$ above the surrounding bilayer, in accordance with literature reports on the expected difference in height between Lo and Ld domains. ${ }^{44,45}$ After membrane topographical characterization, we exposed the SLBs to SEVs. In Fig. $2 \mathrm{~b}$ and c, we report the temporal evolution of the interaction as measured by AFM. We can observe the formation of patches on the surface with the sEV-related domain height roughly protruding $4 \mathrm{~nm}$ above the SLB. Both experiments show colocalization of SEV patches with Lo domains. The AFM images in Fig. 2 are taken on the same SLB spot to follow the fusion kinetics.

The observed interaction of sEVs with the multicomponent membrane is compatible with the preferential docking of sEVs in regions of the SLB where a discontinuity is present (about $2 / 3$ of the total $\mathrm{SEV}$ patches are in close proximity to the discontinuities). ${ }^{46,47}$ Then, SEV lateral diffusion within the membrane seems to be favoured in the liquid disordered phospholipid phase (see also Fig. S6 and S8 $\dagger$ ). As recently discussed in other works, ${ }^{48}$ phase boundaries represent an energetically favourable site for macromolecule interaction with biomembranes, thanks to the thickness mismatch between Ld and Lo domains, where the free energy minimum enables favourable nanoparticles-lipid interactions without the need for large curvature deformations.

Besides, the time evolution of the system topography showed an increased granularity of the liquid ordered domains with liquid/liquid domain boundary lengths increasing upon interaction, as if the line tension across the boundary of the two regions was reduced (see Fig. S6†). A similar effect has never been observed in previous AFM studies we performed on the interaction of biomolecules with membranes with the same composition. ${ }^{44}$ Evidence of Lo domain granularity has instead been reported in the literature in the case of supported membranes with composition similar to ours, following cholesterol depletion ${ }^{49,50}$ as well as molar proportion modification of the lipid components along phase transitions $^{51}$ suggesting that SEV interaction causes a quite immediate membrane component special redistribution. This effect may be attributed to either the depletion of SLB components operated by sEVs or to a fast redistribution of some molecular species from the sEVs, preferentially along the phase borders lowering the tension across the boundary by reducing the hydrophobic mismatch, then generating the observed Lo domain granularity. ${ }^{52,53}$ Our hypothesis is that distinct mixing processes occur: the fast diffusion of some lighter elements, causing these components to laterally migrate along the phase boundaries, and the diffusion of bulkier sEV components that mix within the target membrane and that has been further investigated in the present work. Fig. $2 \mathrm{~b}$ and $\mathrm{c}$ highlight sEVs spreading on the surface by depleting the membrane of its Ld phase portions. We observed a growth in the lateral dimensions of SEV patches versus time. The easiest hypothesis is that sEVs still present in solution fuse with the membrane-fused ones, which behave as preferential docking sites thanks to their enrichment in carbohydrates, known to be involved in membrane-to-membrane adhesion. ${ }^{54,55}$ Such a hypothesis is also supported from the panels (b) and (c) of Fig. 2, where it is evidenced that, 30 minutes after incubation, new SEV patches are detected on the membrane surface. However, we cannot discard the contribution of sequestered lipids from the Ld phase to the lateral spreading of membrane fused-sEV islands. a)

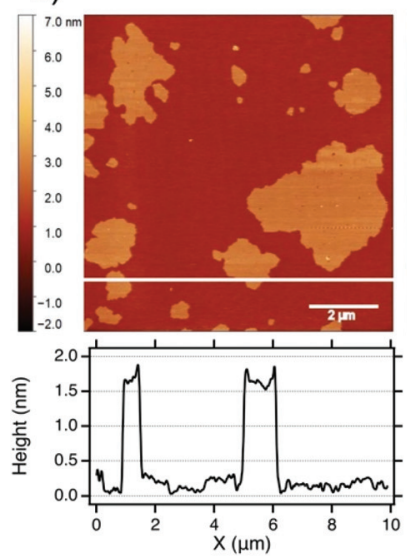

b)
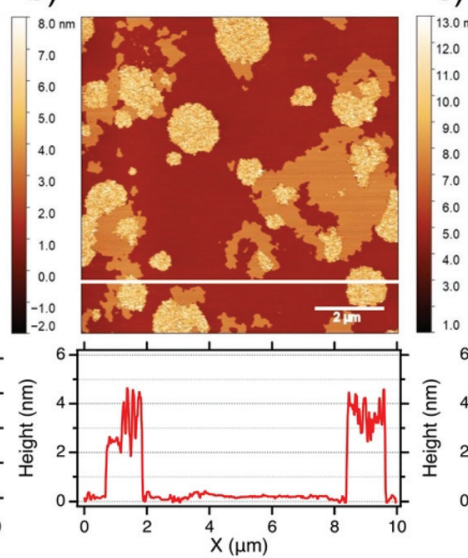

c)
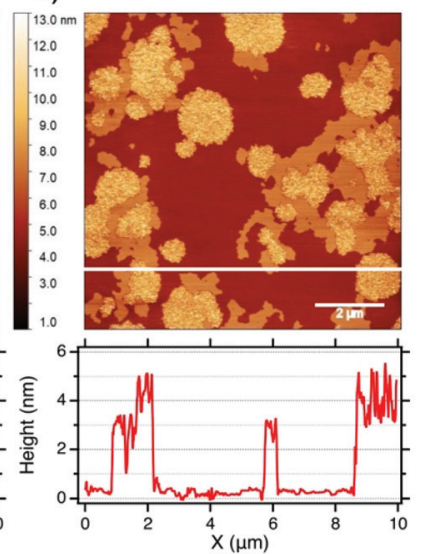

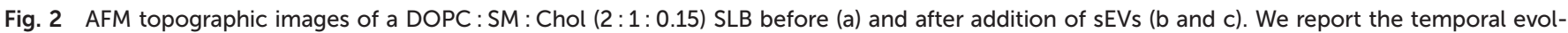

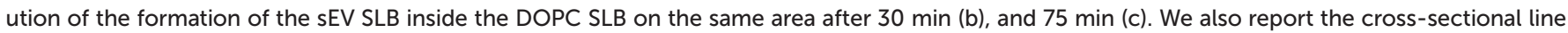

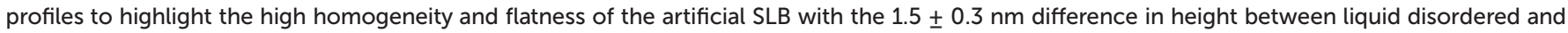
liquid ordered domains (a) and the protrusion of the sEV SLB inside the artificial SLB in (b and c) lateral scale bar is $2 \mu \mathrm{m}$. 
a)
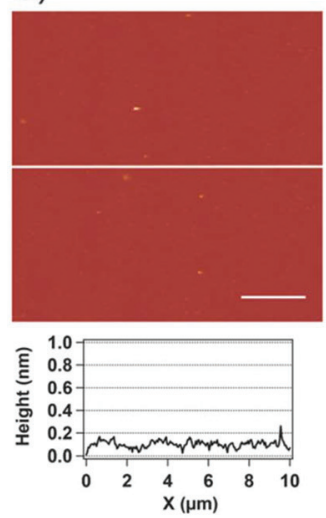

b)
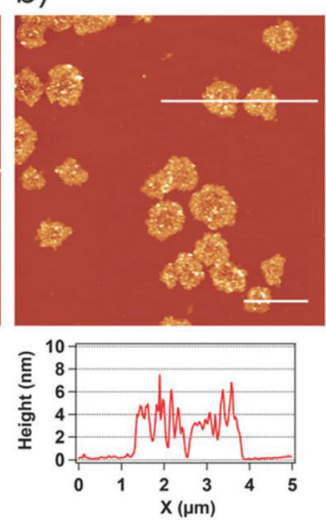

c)
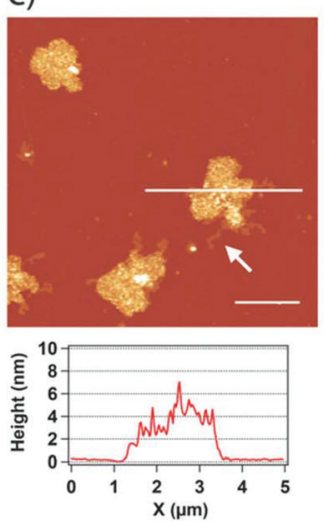

d)

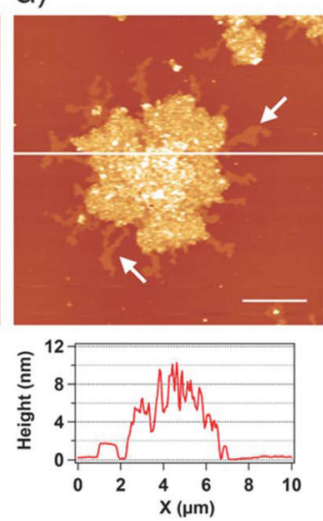

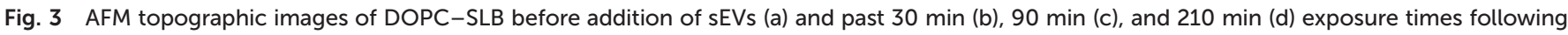

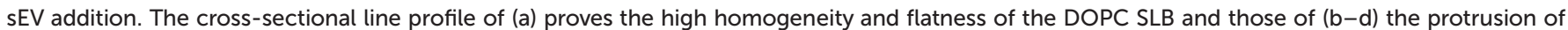

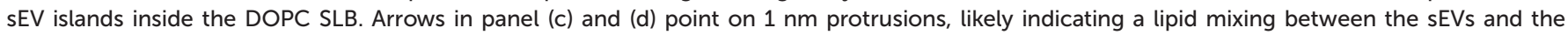
DOPC-SLB. Lateral scale bar is $2 \mu \mathrm{m}$.

In order to highlight the fine structural details of the EVs/ membrane interaction, we performed combined microscopy and neutron scattering studies on less complex membranes optimizing the experimental conditions to obtain NR quantitative results. Since from time resolved AFM imaging we observed that EV lateral diffusion occurs on the membrane liquid phase we moved to single PC component Ld membranes. In Fig. 3a, a representative AFM image and the corresponding line profile of a 1,2-dioleoyl-sn-glycero-3-phosphocholine (DOPC) supported lipid bilayer (SLB) are reported. DOPC SLB presents an extreme homogeneity, an almost defect-free structure and a very small surface roughness $(0.17 \pm 0.05 \mathrm{~nm})$. The bilayer height, as measured from indentation profiles and topographic measurements of partially complete bilayers (see Fig. S3 $\dagger$ ), is $5 \pm 0.2 \mathrm{~nm}$ in agreement with literature reports. ${ }^{56}$ The mixed lipid surface is slightly less defective than the ternary mixture bilayer, in line with the literature. As before, the DOPC SLB topographic image in Fig. 3a is then taken as the time zero snapshot of a time resolved AFM experiment to monitor the bilayer modification upon sEV addition. After 30 minutes following the addition of sEVs, one clearly observes the presence of islands protruding few $\mathrm{nm}$ from the DOPC surface (Fig. 3b). After 30 minutes, there was no trace of intact sEVs, which were rarely identified even at shorter time scales (Fig. S7 $\dagger$ ), a sign of a very fast mixing process. Because of their $\sim 120 \mathrm{~nm}$ diameter in fact intact sEVs when present would be easily recognized by AFM topographic imaging, as shown in Fig. 1a for EVs on mica. On the other hand, the observed protrusion of the islands - namely $3.4 \pm 0.7 \mathrm{~nm}$ - is less pronounced than what was expected from a second lipid bilayer stacking on top of the DOPC bilayer $(5.5 \pm 0.5 \mathrm{~nm}$ was the measured thickness of the DOPC bilayer). Therefore, we interpret this mesoscale heterogeneity to be due to the presence of membrane proteins as well as cargo macromolecules released from the opened EVs in the original DOPC single membrane bilayer. ${ }^{57}$ A similar mesoscale heterogeneity has been observed also in silica-supported EV-derived SLB, ${ }^{58,59}$ there interpreted with the distribution of different portions of the cargo trapped in the SLB.

As a function of SEV exposure time (Fig. 3c and d), the islands grow in lateral dimensions, until they coalesce into larger islands (of up to 3-4 micrometers in diameter after 4 hours of exposure time). Upon coalescence, the 3D profile of islands changes, in a way compatible with the accumulation of biomolecules of SEV origin on the patches already formed. The nucleated islands serve in fact as docking points for new incoming sEVs, which can open on them either face-up and release their content on the island, or face-down favouring the accumulation of cargo biomolecules below the original sEV bilayer, in agreement with what has been reported by Montis and coworkers. ${ }^{59}$ From the growth evolution of the sEVenriched patches derived from the AFM images in Fig. 3, we then speculate the formation of a single sEV-related bilayer showing a (partial) mixing of the vesicles with the SLB as a preliminary step towards complete fusion of the SEVs with the membrane itself. Lipid mixing is also supported by the appearance of a new, likely lipid phase around the SEV patches, which protrudes by about $1 \mathrm{~nm}$ (Fig. 3d), after 4 hours exposure time. Such a phase might be assigned to the partial diffusion of sEVs' lipids in the DOPC layer and/or to a misalignment of DOPC molecules due to the presence of proteins or other molecules diffusing under the bilayer. A similar mechanism of membrane fusion has been also hypothesized, based on combined atomic force and fluorescence microscopy experiments, for proteoliposomes interacting with the SLB, ${ }^{60}$ in accordance with our findings. In order to verify the presence of proteins in the sEV-related lipid phase, we incubated sEVs treated with proteinase $\mathrm{K}$ according to the protocol of Skliar et al. ${ }^{61}$ to a DOPC SLB for $90 \mathrm{~min}$, as shown in Fig. 3(b), and monitored the morphology of the system with AFM. From Fig. S4, $\dagger$ we observe the presence of patches with lateral dimensions comparable to those originated from non-treated 
sEVs. However, the average height is now roughly the same as that of the DOPC layer, although with a sensibly increased roughness. Altogether these findings indicate that (i) the sEVs completely open on the SLB; (ii) the topographical heterogeneity observed in Fig. 3 is attributable to SEV proteins; (iii) upon addition of sEVs, a mixed SLB forms in which the lipid components of the EV tend to remain segregated/intercalated in the DOPC SLB, suggesting a low influence of the proteic components in preserving segregation. Moreover, comparing the temporal evolution of the coverage of the sEV-related portions of the pure DOPC and DOPC:SM : Chol artificial bilayers, we observe a first-order Langmuir absorption behavior in both cases (Fig. S6†), with a similar kinetic constant but with a larger final coverage in the case of the mixed composition SLB. This is probably ascribable to sEVs' higher affinity towards the mixed membrane, due to the preferential docking at the edges of the rafts.

Yet, a full understanding of the fusion mechanism would be required to discriminate the possible fusion asymmetries occurring in the two membrane leaflets. To further clarify the interaction of SEVs with the PC lipid bilayer, and investigate the extent of fusion, that is if fusion involved only the external target membrane leaflet or the whole membrane, we performed NR and SANS measurements. NR allowed investigation of the transverse structure of a single supported deuterated phospholipid (d54-DMPC) bilayer in the fluid phase before and after the interaction with sEVs. The phospholipid membrane was prepared by vesicle fusion. The DMPC was deuterated in order to exploit the proton-deuteron (actually hydrogen-deuterium, H-D) contrast difference in neutron scattering, with the Scattering Length Density (SLD) of deuterated lipids being around $7 \times 10^{-6} \AA^{-2}$, that of protiated lipids -0.3 $\times 10^{-6} \AA^{-2}$ and that of EVs around $2 \times 10^{-6} \AA^{-2}$. The use of deuterated lipids for the target membrane allowed the maximization of the visibility of EVs after mixing, and obtaining the peculiar information about the final complex system, otherwise difficult to obtain. This approach is consolidated to monitor the interaction of macromolecules, ${ }^{62-64}$ as we did with alpha-synuclein in interactions with artificial bilayers, ${ }^{65}$ and as was similarly exploited by Ghosh et al. to study synaptic vesicles. ${ }^{66}$ First, the neutron reflectivity of the bulk deuterated bilayer was recorded, then, a solution containing isolated EVs was added to the cell with the same sEV/lipid ratio used in the AFM experiments and the NR was recorded again. By comparing the reflectivity profiles, one expects to see how the H-rich sEVs penetrate the deposited D-rich bilayer. The measured reflectivity profiles together with the simultaneous best-fits are reported in Fig. $\mathrm{S} 2 \dagger$ (best fit parameters in Table S2 $\uparrow$ ), while the relative SLD profiles are shown in Fig. 4.

The SLD profile analysis shows an overall increase in the thickness of the membrane upon SEV addition and an overall modification of the SLD profile across the membrane. The increased membrane thickness of $5.4 \pm 0.1 \mathrm{~nm}$ (from $4.1 \pm$ $0.1 \mathrm{~nm}$ in the bulk bilayer) is an intermediate value between that of a pure phospholipid membrane and a sEV-based supported membrane $(6.9 \mathrm{~nm})$, as reported in Fig. $2 \mathrm{~b}$. We also

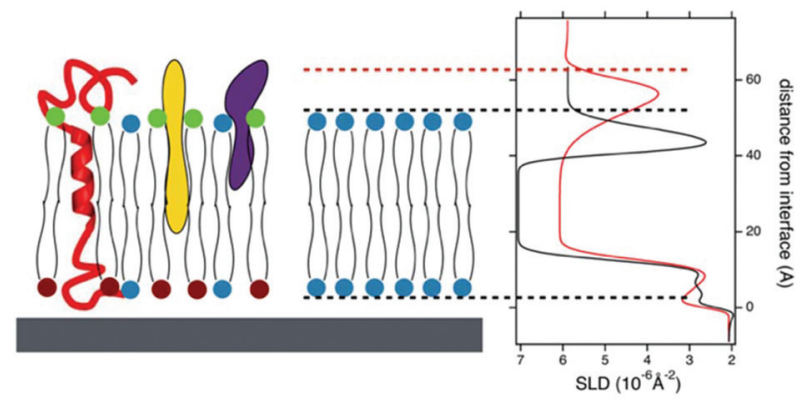

Fig. 4 Scattering length density profiles of a deuterated PC SLB before (black) and after sEVs interaction (red) obtained by neutron reflectivity with a pictorial sketch to help in SLD profile interpretation. Measured reflectivity profiles and best fit parameters are reported in the ESI. $\dagger$

observed a decrease in the SLD value of the membrane from $7.1 \pm 0.2 \times 10^{-6} \AA^{-2}$ to $6.1 \pm 0.2 \times 10^{-6} \AA^{-2}$, consistent with the fusion of the H-containing sEVs into the deuterated membrane with about $20 \%$ volume penetration. This experiment demonstrates the high fusogenic ability of EVs with the lipidonly target membrane. Moreover, the fusion occurs "transmembrane", i.e. in the entire thickness of the bilayer, involving not only the external, but also the inner layer (with respect to the solid support) of the bilayer. However, the final membrane profile becomes slightly asymmetric (see Fig. 4). This asymmetry may reflect an actual uneven distribution or rough feature of SEV fusion with the bilayer, even if a $0.5 \mathrm{~nm}$ thick water layer was found between the membrane and the silicon support, and the lack of space may hinder the fusion mechanisms and induce asymmetric fusion. In addition, we cannot exclude the influence of the silicon support, generally negatively charged in an aqueous environment, in impeding the most extended hydrophilic molecular portions to position themselves in the inner leaflet.

To dispel these questions, we performed mixing experiments in bulk applying SANS to study the structural details of mixed solutions containing unilamellar phospholipid vesicles and sEVs in different proportions (details in the Materials and methods section). Again, deuterated phospholipid vesicles were used to benefit the $\mathrm{H}-\mathrm{D}$ contrast difference and to reduce the incoherent background due to the $\mathrm{H}$ atoms. Fig. 5(a) shows the summary of the SANS results on the low, medium and high concentration vesicle-to-EV mixtures of number ratios $15000: 1 ; 3000: 1$ and 2700:1, respectively. The measured intensities can be compared with those of the pure phospholipid vesicles (red circles) and pure sEVs (black circles) as well as their weighed sum in the respective proportions (dotted lines). Panel (c) shows the SLD distributions derived from the fit of the SANS data. Despite the low molar proportion of SEVs with respect to target vesicles, the H-bringing sEV scattering intensity is highlighted within the D-based phospholipid target membranes, allowing for a detailed structural investigation of the mixed systems.

In Fig. 5a, the shapes of the reconstructed (by linear combination of the two contributions) and the measured curves are 


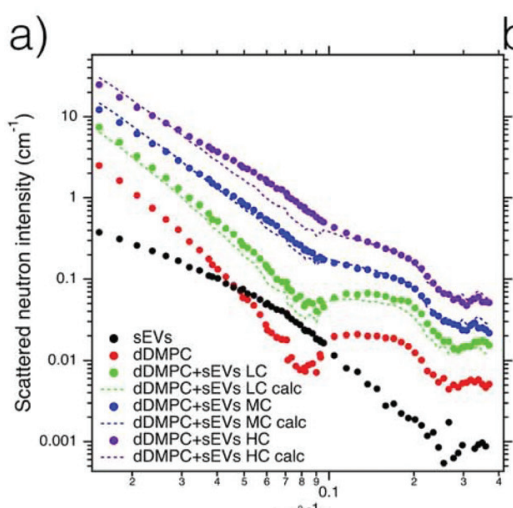

$\mathrm{q}\left(\AA^{-1}\right)$

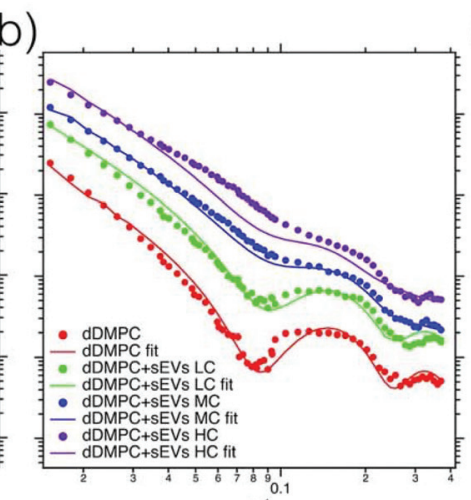

$q\left(\AA^{-1}\right)$
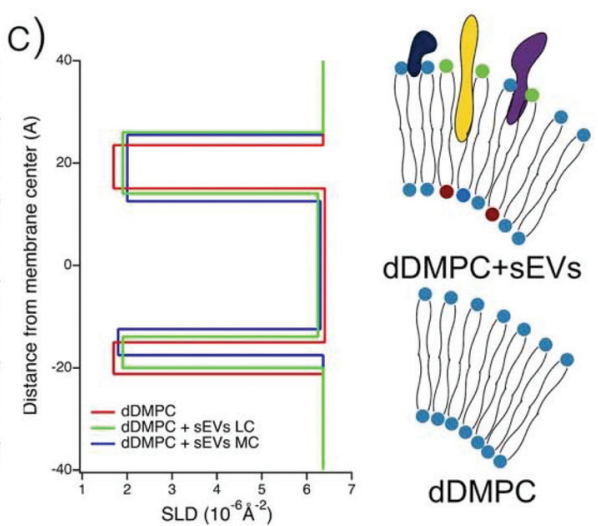

Fig. 5 SANS measurements on deuterated PC unilamellar vesicles at $20 \mathrm{mg} \mathrm{mL}^{-1}$ (red circles), sEVs at a concentration of $2 \times 10^{9} \mathrm{EVs}$ in $100 \mu \mathrm{L}$ (black circles) and the mixed systems at low (low concentration, LC, green circles), medium (medium concentration, MC, blue circles) and high (high concentration, HC, violet circles) relative proportions, see text. (a) Dotted lines represent the weighed sum of the spectra of the two pure components (phospholipid vesicles and SEVs) of appropriate proportions. (b) Continuous lines are the fits of the experimental curves with a threelayered vesicular model, parameters in the ESI. $\dagger$ (c) SLD profiles extracted from fits to the SANS data (see the ESI $\dagger$ ).

different, indicating a non-negligible interaction between the constituents. Notably, the residual Bragg multilayer signal at $0.1 \AA^{-1}$ detected for the PC target vesicles (red circles) was lost in the mixed systems even at the lowest SEV content, indicating that fusion events remove the residual multi-lamellarity of the original vesicles. For medium and high SEV concentration, the experimental scattering profiles cross the reconstructed ones, indicating that a change in the SLD of the objects occurred. This suggests a decrease in the overall size of the mixed systems with respect to the original PC vesicles, as shown by the lower intensity at low $q$ vectors.

Hypothesizing that the interaction leads to a unique mixed vesicle distribution, we tentatively analyzed the data by modelling the system with the form factor of a (three) layered vesicular system (outer hydrophilic, hydrophobic and inner hydrophilic layers) with a water core. This simplified model seems to work well for systems with null or low SEV content and becomes progressively less accurate for systems with larger SEV concentration. Best fit results and parameters are reported in Fig. 5b and Table S3, $\dagger$ while the SLD profiles of the single bilayer obtained by the data best fits are shown in Fig. $5 \mathrm{c}$. Data analysis is consistent with the lowering of the SLD of the hydrophobic portion, indicating that the mixing involves both membrane leaflets, in accordance with the NR results. Moreover, the thickness of the hydrophobic portion appears to decrease upon sEV mixing, while the thickness of the external polar portion increases at increasing sEV content. Results on thickness alteration agree with both AFM and NR findings. Furthermore, the analysis is consistent with the formation of an asymmetric membrane upon EV mixing. The external hydrophilic layer becomes more extended after fusion, as if the largest membrane components belonging to sEVs were not included in the inner membrane leaflet of the final vesicular system, but only fused to the outer one, as already observed by NR and depicted in Fig. 4.
These findings unveil a mixing mechanism distinct from total fusion, in which rather lipids and smaller molecules might fuse and eventually flip into the inner membrane leaflets, while large proteins bringing important hydrophilic portions could eventually reside in the outer membrane leaflet. This asymmetry is kept stable for a long time (2 days of measurements), suggesting that the flip-flop is not energetically favored. When considering this limit to total fusion, it is important to recall that so far we have been dealing with simple models of target membranes. Rather, in natural biological membranes, the lateral complexity related to the occurrence of ordered domains of different lipids and to the presence of membrane proteins may play a significant role in fusion mechanisms. The importance of our findings and our approach, which can in principle be translated to other systems of growing complexity and/or sEVs of different origins, resides in the capability to in principle disentangle the different contributions to the fusion mechanisms, addressing them one at a time, with the ultimate goal of controlling single molecular components to optimize cell-to-cell communication through sEVs, even in sight of cargo release chances for therapeutic purposes.

\section{Conclusions}

Here, we provided a molecular description of the interaction of sEVs with SLBs as model plasma membrane systems. We showed that AFM morphological analysis can be profitably combined with neutron scattering-based structural investigation to successfully elucidate the molecular mechanisms of interaction of sEVs with membranes, from which SEV cargo release may depend. In particular, we demonstrated the sEV propensity to fuse with the model membranes through a not total fusion mechanism, involving different diffusion events. 
Also, we highlighted the critical role of phase borders to promote sEV fusion. The use of variable composition SLBs as model systems to mimic cell membranes will be particularly useful in studies of EVs-cell uptake, in which concurrent multiple uptake channels such as lipid-raft mediated endocytosis, surface binding and membrane fusion might be involved, ${ }^{16}$ allowing the disentanglement of the various contributions addressing them one at a time. These different mechanisms are in fact particularly difficult to distinguish in a complex cellular environment. ${ }^{67-69}$ Knowledge acquired from model systems on the prevalent uptake mechanism of distinct EV subpopulations and on the specific membrane subdomain involved will allow the design of specific strategies to act on the defined internalization channel and/or membrane compartment to block or favour cell EV interactions and cargo release to target cells in the context of a given disease. The approach shown here can be extended to convey incremental complexity, adding, for example, glycosphingolipid and membrane proteins to the model lipid bilayers. We strongly believe that our approach combined with data on the specific biological function of each EV subpopulation as retrieved by standard functional assays will prove useful to select the crucial molecular aspects of EV internalization by cells. Such information might be then exploited to develop novel devices based on standard readout platforms, e.g. fluorescence, mechanical and/or electrochemical readout, to screen EVs for particular properties to predict their functionality, thus revolutionizing the way how EVs may be used in diagnostics and hopefully become useful in therapies.

\section{Author contributions}

F. P., V. R., L. C. and P. P. conceived and planned the experiments. F. P., B. Se., and P. P. performed the atomic force microscopy experiments and analysed the data. F. P., V. R., P. P., H. A. and B. Sa. performed the SAXS experiments. L. A., V. R., F. P. and P. P. performed the SANS experiments. L. B., D. G. M., V. R., F. P., and P. P. performed the neutron reflectometry experiments. V. R., P. B. and P. P. analysed the scattering and reflectrometry data. K. P., M. M., M. G. and E. R. contributed to EV isolation and molecular characterization. P. P. and L. C. took the lead in writing the manuscript. All authors provided critical feedback and helped shape the research, analysis and manuscript.

\section{Conflicts of interest}

There are no conflicts to declare.

\section{Acknowledgements}

The authors acknowledge funding from the European Regional Development Fund Interreg V-A Italia-Austria 2014-2020 (EXOTHERA ITAT1036) and the Department of
Medical Biotechnologies and Translational Medicine of the Università degli Studi di Milano for grant PSR 2019 to VR and PB. We acknowledge CERIC-ERIC proposal grant n. 20187082 to perform measurements at the Austrian SAXS beamline of Elettra Sincrotrone Trieste S.C.p.A. and at GINA and SANS-YS instruments of the Budapest Neutron Center. High purity cleaning of the Si block surface by the staff of the BioMEMS group of Inst. Tech. Physics and Mater. Sci., Budapest is gratefully acknowledged. We gratefully acknowledge the assistance of H. M. Binder and D. Auer (PMU Salzburg) for flow cytometry analyses, and A. Desgeorges (PMU Salzburg) for NTA analyses.

\section{References}

1 M. Mathieu, L. Martin-Jaular, G. Lavieu and C. Thery, Nat. Cell Biol., 2019, 21, 9.

2 C. Théry, et al., J. Extracell. Vesicles, 2020, 7, 1.

3 A. Thompson, E. Gray, S. Heman-Ackah, I. Mager, S. El Andaloussi, K. Talbot, M. J. Wood and M. R. Turner, Nat. Rev. Neurol., 2016, 12, 346.

4 N. Kosaka, A. Kogure, T. Yamamoto, F. Urabe, W. Usuba, M. Prieto-Villa and T. Ochiya, Exp. Mol. Med., 2019, 51, 31.

5 E. Buzas, B. György, B. Nagy, G. A. Falus and S. Gay, Nat. Rev. Rheumatol., 2014, 10, 356.

6 C. Boulanger, X. Loyer, P. Rautou and N. Amabile, Nat. Rev. Cardiol., 2017, 14, 259.

7 S. Fais, L. O'Driscoll, F. E. Borras, E. Buzas, G. Camussi, F. Cappello, J. Carvalho, A. Cordeiro da Silva, H. Del Portillo, S. El Andaloussi, T. Ficko Trček, R. Furlan, A. Hendrix, I. Gursel, V. Kralj-Iglic, B. Kaeffer, M. Kosanovic, M. E. Lekka, G. Lipps, M. Logozzi, A. Marcilla, M. Sammar, A. Llorente, I. Nazarenko, C. Oliveira, G. Pocsfalvi, L. Rajendran, G. Raposo, E. Rohde, P. Siljander, G. van Niel, M. H. Vasconcelos, M. Yáñez-Mó, M. L. Yliperttula, N. Zarovni, A. Bedina Zavec and B. Giebel, ACS Nano, 2016, 10, 3886-3899.

8 D. E. Murphy, O. G. Jong, M. Brouwer, M. J. Wood, G. Lavieu, R. M. Schiffelers and P. Vader, Exp. Mol. Med., 2019, 51, 32.

9 S. Sharma, M. LeClaire, J. Wohlschlegel and J. Gimzewski, Sci. Rep., 2020, 10, 13327.

10 F. Royo, C. Théry, J. M. Falcón-Pérez, R. Nieuwland and K. W. Witwer, Cells, 2020, 9, 1955.

11 F. Perissinotto, B. Senigagliesi, L. Vaccari, M. Pachetti, F. D’Amico, H. Amenitsch, B. Sartori, K. Pachler, M. Mayr, M. Gimona, E. Rohde, F. Caponnetto, D. Cesselli, L. Casalis and P. Parisse, Adv. Biomembr. Lipid Self-Assem., 2020, 32, 157.

12 F. A. W. Coumans, A. R. Brisson, E. I. Buzas, F. DignatGeorge, E. E. E. Drees, S. El-Andaloussi, C. Emanueli, A. Gasecka, A. Hendrix, A. F. Hill, R. Lacroix, Y. Lee, T. G. van Leeuwen, N. Mackman, I. Mäger, J. P. Nolan, E. van der Pol, D. M. Pegtel, S. Sahoo, P. R. M. Siljander, G. Sturk, O. de Wever and R. Nieuwland, Circ. Res., 2017, 120, 1632. 
13 L. Paolini, A. Zendrini and A. Radeghieri, Biomarkers Med., 2018, 12, 383.

14 L. Margolis and Y. Sadovsky, PLos Biol., 2019, 17, e3000363. 15 M. I. Ramirez, M. G. Amorim, C. Gadelha, I. Milic, J. A. Welsh, V. M. Freitas, M. Nawaz, N. Akbar, Y. Couch, L. Makin, F. Cooke, A. L. Vettore, P. X. Batista, R. Freezor, J. A. Pezuk, L. Rosa-Fernandes, A. C. O. Carreira, A. Devitt, L. Jacobs, I. T. Silva, G. Coakley, D. N. Nunes, D. Carter, G. Palmisano and E. Dias-Neto, Nanoscale, 2018, 10, 881906.

16 K. C. French, M. A. Antonyak and R. A. Cerione, Semin. Cell Dev. Biol., 2017, 67, 48.

17 E. I. Buzas, E. Á. Tóth, B. W. Sódar and K. É. Szabó-Taylor, Semin. Immunopathol., 2018, 40, 453.

18 L. Mulcahy, R. C. Pink and D. R. Carter, J. Extracell. Vesicles, 2014, 3, 24641.

19 K. Umezaki, S. Sawada, S. Mukai, Y. Sasaki, N. Harada, H. Shiku and K. Akiyoshi, Sci. Rep., 2016, 6, 21933.

20 M. Yáñez-Mó, P. R.-M. Siljander, Z. Andreu, A. Bedina Zavec, F. E. Borràs, E. I. Buzas, K. Buzas, E. A. Casal, F. Cappello, J. Carvalho, E. Colás, A. Cordeiro-da Silva, S. Fais, J. M. Falcon-Perez, I. M. Ghobrial, B. Giebel, M. Gimona, M. Graner, I. Gursel, M. Gursel, N. H. H. Heegaard, A. Hendrix, P. Kierulf, K. Kokubun, M. Kosanovic, V. Kralj-Iglic, E.-M. Krämer-Albers, S. Laitinen, C. Lässer, T. Lener, E. Ligeti, A. Linē, G. Lipps, A. Llorente, J. Lötvall, M. Manček-Keber, A. Marcilla, M. Mittelbrunn, I. Nazarenko, E. N. M. Nolte-'t Hoen, T. A. Nyman, L. O'Driscoll, M. Olivan, C. Oliveira, É. Pállinger, H. A. del Portillo, J. Reventós, M. Rigau, E. Rohde, M. Sammar, F. Sánchez-Madrid, N. Santarém, K. Schallmoser, M. Stampe Ostenfeld, W. Stoorvogel, R. Stukelj, S. G. Van der Grein, M. H. Vasconcelos, M. H. M. Wauben and O. De Wever, J. Extracell. Vesicles, 2015, 4, 1.

21 F. Caponnetto, I. Manini, M. Skrap, T. Palmai-Pallag, C. Di Loreto, A. P. Beltrami, D. Cesselli and E. Ferrari, Nanomedicine, 2016, 13, 1011.

22 T. Tian, Y. L. Zhu, F. H. Hu, Y. Y. Wang, N. P. Huang and Z. D. Xiao, J. Cell. Physiol., 2013, 228, 1487.

23 K. Wang, L. Zhou, J. Li, W. Liu, Y. Wei, Z. Guo, C. Fan, J. Hu, B. Li and L. Wang, Nano Lett., 2020, 20(9), 63136319.

24 A. E. Saliba, I. Vonkova and A. C. Gavin, Nat. Rev. Mol. Cell Biol., 2015, 16, 653.

25 V. Rondelli, E. Del Favero, P. Brocca, G. Fragneto, M. Trapp, L. Mauri, M. G. Ciampa, G. Romani, C. J. Braun, L. Winterstein, I. Schroeder, G. Thiel, A. Moroni and L. Cantu', Biochim. Biophys. Acta, 2018, 1862, 1742.

26 V. Rondelli, P. Brocca, S. Motta, M. Messa, L. Colombo, M. Salmona, G. Fragneto, L. Cantù and E. Del Favero, Sci. Rep., 2016, 6, 20977.

27 L. Colombo, A. Gamba, L. Cantù, M. Salmona, F. Tagliavini, V. Rondelli, E. Del Favero and P. Brocca, Biophys. Chem., 2017, 229, 11.
28 M. Y. Konoshenko, E. A. Lekchnov, A. V. Vlassov and P. P. Laktionov, BioMed Res. Int., 2018, 2018, 8545347.

29 O. P. B. Wiklander, R. B. Bostancioglu, J. A. Welsh, A. M. Zickler, F. Murke, G. Corso, U. Felldin, D. W. Hagey, B. Evertsson, X.-M. Liang, M. O. Gustafsson, D. K. Mohammad, C. Wiek, H. Hanenberg, M. Bremer, D. Gupta, M. Björnstedt, B. Giebel, J. Z. Nordin, J. C. Jones, S. El Andaloussi and A. Görgens, Front. Immunol., 2018, 9, 1326.

30 J. Lotvall, A. F. Hill, F. Hochberg, E. I. Buzas, D. di Vizio, C. Gardiner, Y. S. Gho, I. V. Kurochkin, S. Mathivanan, P. Quesenberry, et al., J. Extracell. Vesicles, 2014, 3, 26913.

31 P. Parisse, I. Rago, L. Ulloa Severino, F. Perissinotto, E. Ambrosetti, P. Paoletti, M. Ricci, A. P. Beltrami, D. Cesselli and L. Casalis, Eur. Biophys. J., 2017, 46, 813.

32 N. Sebaihi, B. De Boeck, Y. Yuana, R. Nieuwland and J. Péter, Meas. Sci. Technol., 2017, 28, 034006.

33 R. Sorkin, R. Huisjes, F. Bošković, D. Vorselen, S. Pignatelli, Y. Ofir-Birin, J. K. Freitas Leal, J. Schiller, D. Mullick, W. H. Roos, G. Bosman, N. Regev-Rudzki, R. M. Schiffelers and G. J. L. Wuite, Small, 2018, 14, 1801650.

34 D. P. Romancino, V. Buffa, S. Caruso, I. Ferrara, S. Raccosta, A. Notaro, Y. Campos, R. Noto, V. Martorana, A. Cupane, A. Giallongo, A. d'Azzo, M. Manno and A. Bongiovanni, Biochim. Biophys. Acta, 2018, 1862, 2879.

35 A. Sarra, A. Celluzzi, S. P. Bruno, C. Ricci, S. Sennato, M. G. Ortore, S. Casciardi, F. Del Chierico, P. Postorino, F. Bordi and A. Masotti, Front. Microbiol., 2020, 11, 290.

36 S. Castorph, D. Riedel, L. Arleth, M. Sztucki, R. Jahn, M. Holt and T. Salditt, Biophys. J., 2010, 98, 1200.

37 I. Rodriguez-Loureiro, V. M. Latza, G. Fragneto and E. Schneck, Biophys. J., 2018, 114, 1624.

38 S. Waldie, T. K. Lind, K. Browning, M. Moulin, M. Haertlein, V. T. Forsyth, A. Luchini, G. A. Strohmeier, H. Pichler, S. Maric and M. Cárdenas, Langmuir, 2018, 34, 472.

39 G. M. Cooper, The Cell: A Molecular Approach, Sinauer Associates, Sunderland (MA), 2nd edn, 2000.

40 H. P. Wacklin, Curr. Opin. Colloid Interface Sci., 2010, 15, 445-454.

41 Y. Gerelli, A. de Ghellinck, J. Jouhet, V. Laux, M. Haertleina and G. Fragneto, Biol. Crystallogr., 2014, 70, 3167.

42 G. Zaccai, J. K. Blasie and B. P. Schoenborn, Proc. Natl. Acad. Sci. U. S. A., 1975, 72, 376.

43 N. Bezlyepkina, R. S. Gracia, P. Shchelokovskyy, R. Lipowsky and R. Dimova, Biophys. J., 2013, 104, 1456.

44 F. Perissinotto, C. Stani, E. De Cecco, L. Vaccari, V. Rondelli, P. Posocco, D. Scaini, G. Legname, P. Parisse and L. Casalis, Nanoscale, 2020, 12, 7631.

45 G. G. Shipley, L. S. Avecilla and D. M. Small, J. Lipid Res., 1974, 15, 124.

46 R. J. Molotkovsky, V. V. Alexandrova, T. R. Galimzyanov, I. Jiménez-Munguía, K. V. Pavlov, O. V. Batishchev and S. A. Akimov, Int. J. Mol. Sci., 2018, 19, 1483.

47 J. K. Sheavly, J. A. Pedersen and R. C. Van Lehn, Nanoscale, 2019, 11, 2767. 
48 A. Ridolfi, L. Caselli, C. Montis, G. Mangiapia, D. Berti, M. Brucale and F. Valle, J. Microsc., 2020, 280, 194.

49 J. C. Lawrence, D. E. Saslowsky, J. M. Edwardson and R. M. Hederson, Biophys. J., 2003, 84, 1827.

50 J. Zhong, C. Yang, W. Zheng, L. Huang, Y. Hong, L. Wang and Y. Sha, Biophys. J., 2009, 96, 4610.

51 N. K. Khadka, C. S. Ho and J. Pan, Langmuir, 2015, 31, 12417.

52 P. I. Kuzmin, S. A. Akimov, Y. A. Chizmadzhev, J. Zimmerberg and F. S. Cohen, Biophys. J., 2005, 88, 1120.

53 S. S. Iyer, M. Tripathy and A. Srivastava, Biophys. J., 2018, $115,117$.

54 S. Hakomori, Pure Appl. Chem., 1991, 63, 473-482.

55 R. D. Cummings, Glycoconjugate J., 2019, 36, 241-257.

56 S. Attwood, Y. Choi and Z. Leonenko, Int. J. Mol. Sci., 2013, 14, 3514 .

57 N. Galvanetto, Biochim. Biophys. Acta, Biomembr., 2018, 1860, 2532.

58 C. Montis, S. Busatto, F. Valle, A. Zendrini, A. Salvatore, Y. Gerelli, D. Berti and P. Bergese, Adv. Biosyst., 2018, 2, 1700200.

59 C. Montis, A. Salvatore, F. Valle, L. Paolini, F. Carlà, P. Bergese and D. Berti, J. Colloid Interface Sci., 2020, 570, 340.

60 R. Tero, K. Fukumoto, T. Motegi, M. Yoshida, M. Niwano and A. Hirano-Iwata, Sci. Rep., 2017, 7, 17905.

61 M. Skliar, V. S. Chernyshev, D. M. Belnap, G. V. Sergey, S. M. Al-Hakami, P. S. Bernard, I. J. Stijleman and
R. Rachamadugu, Biochem. Biophys. Res. Commun., 2018, 6, 1055.

62 V. Rondelli, E. Del Favero, P. Brocca, G. Fragneto, M. Trapp, L. Mauri, M. G. Ciampa, G. Romani, C. J. Braun, L. Winterstein, I. Schroeder, G. Thiel, A. Moroni and L. Cantu, Biochim. Biophys. Acta, Gen. Subj., 2018, 1862, 1742.

63 V. Rondelli, P. Brocca, S. Motta, M. Messa, L. Colombo, M. Salmona, G. Fragneto, L. Cantù and E. Del Favero, Sci. Rep., 2016, 6, 20997.

64 V. Rondelli, M. Salmona, L. Colombo, G. Fragneto, G. C. Fadda, L. Cantu' and E. Del Favero, Int. J. Mol. Sci., 2020, 21, 8295.

65 F. Perissinotto, V. Rondelli, P. Parisse, N. Tormena, A. Zunino, L. Almásy, D. G. Merkel, L. Bottyán, Sz. Sajti and L. Casalis, Biophys. Chem., 2019, 255, 106272.

66 S. K. Ghosh, S. Castorph, O. Konovalov, R. Jahn, M. Holt and T. Salditt, New J. Phys., 2010, 12, 105004.

67 M. Axmann, E. Sezgin, A. Karner, J. Novacek, M. D. Brodesser, C. Röhrl, J. Preiner, H. Stangl and B. Plochberger, Nano Lett., 2019, 19, 2562-2567.

68 B. Plochberger, M. Axmann, C. Röhrl, J. Weghuber, M. Brameshuber, B. K. Rossboth, S. Mayr, R. Ros, R. Bittman, H. Stangl and G. J. Schütz, Atherosclerosis, 2018, 277, 53.

69 C. Williams, R. Pazos, F. Royo, E. González, M. RouraFerrer, A. Martinez, J. Gamiz, N.-C. Reichardt and J. M. Falcón-Pérez, Sci. Rep., 2019, 9, 1192. 